\title{
Small-radius clean and metal-doped boron carbide nanotubes: A density functional study
}

\author{
Olena Ponomarenko, M. W. Radny,* and P. V. Smith \\ School of Mathematical and Physical Sciences, The University of Newcastle, Callaghan, New South Wales, Australia, 2308
}

(Received 24 November 2005; revised manuscript received 3 July 2006; published 20 September 2006)

\begin{abstract}
We present the results of ab initio density functional theory calculations of the atomic and electronic structure of small radius boron carbide nanotubes, and the effects of doping these tubes with $\mathrm{Li}$ and $\mathrm{Cu}$ atoms. We have found that undoped narrow tubes are more energetically favorable than the corresponding boron carbide strips. The effects of doping on the atomic and electronic structure of these tubes have been found to be dependent on the size and symmetry of the tubes, and the type of dopant atoms.
\end{abstract}

DOI: 10.1103/PhysRevB.74.125421

PACS number(s): 61.46.- w, 73.22.-f

\section{INTRODUCTION}

A decade has passed since the discovery of carbon nanotubes, ${ }^{1}$ and the list of different materials from which nanotubular structures can be formed has been rapidly expanding. Noncarbon nanotubes such as $\mathrm{BN}$, (Ref. 2). $\mathrm{BC}_{2} \mathrm{~N}$, (Ref. 3), $\mathrm{B}_{\mathrm{x}} \mathrm{C}_{\mathrm{y}} \mathrm{N}_{\mathrm{z}}$, (Ref. 4), $\mathrm{BC}_{3}$, (Ref. 3), $\mathrm{CN}_{\mathrm{x}}$, (Ref. 5), $\mathrm{GaN}$, (Ref. 6), $\mathrm{WS}_{2}$, (Ref. 7), $\mathrm{MoS}_{2}$, (Ref. 7), $\mathrm{NiCl}_{2}$, (Ref. 8), $\mathrm{SnS}_{2}$, (Ref. 9), Te, (Ref. 10), Bi, (Ref. 11), $\mathrm{NbSe}_{2}$, (Ref. 12) and $\mathrm{TiSe}_{2}$ (Ref. 13) have been observed experimentally and studied theoretically. It has been suggested that the ability of a material to exist in a layered three-dimensional bulk structure may be a prerequisite for the formation of corresponding nanotubular structures. ${ }^{14}$ This has prompted a number of theoretical studies focused on the physical and chemical properties of hypothetical nanotubes made from various layered materials. ${ }^{15-24}$ Theoretical studies have also been conducted on nanotubes based on superconducting materials such as $\mathrm{NbSe}_{2}$, (Ref. 16), $\mathrm{Ca}\left(\mathrm{Al}_{\mathrm{x}} \mathrm{Si}_{1-x}\right)_{2}$, (Ref. 19) and $\mathrm{Sr}\left(\mathrm{Ga}_{x} \mathrm{Si}_{1-x}\right)_{2}$ (Ref. 19). This includes $\mathrm{MB}_{2}$ materials $[M=\mathrm{Mg}, \mathrm{Al}, \mathrm{Sc}, \mathrm{Ti}$, (Ref. 18), Al, (Ref. 25) Be (Ref. 26)] that are related to the recently discovered layered superconductor $\mathrm{MgB}_{2}$ (transition temperature $\left.T_{\mathrm{C}} \sim 40 \mathrm{~K}\right) .{ }^{27}$ Research on the noncarbon $\mathrm{MB}_{2}$-type nanotubes has been stimulated mainly by the theoretical prediction that introducing curvature into superconducting layered systems might lead to an increase of $T_{\mathrm{C}}$ in cylindrical $\mathrm{MB}_{2}$ wires. $^{28}$

In this paper we study, using ab initio density functional theory (DFT), the properties of clean boron carbide (BC) nanotubes, and $\mathrm{BC}$ nanotubes doped with $\mathrm{Li}$ and $\mathrm{Cu}$ atoms. As layered semiconductors, $\mathrm{CuBC}$ and $\mathrm{LiBC}$ are isovalent with, and structurally similar to, the medium-temperature superconductor $\mathrm{MgB}_{2}$. Both consist of hexagonal $\mathrm{BC}$ layers with alternating boron and carbon atoms. It has been predicted that hole doping of $\mathrm{LiBC}$ would lead to superconductivity of this material. ${ }^{29}$ Calculations by Mehl et al. ${ }^{30}$ have shown that the full replacement of the $\mathrm{Mg}$ atoms by $\mathrm{Cu}$ in $\mathrm{MgB}_{2}$, and the partial replacement of boron atoms by carbon, to form a $\mathrm{Cu} \mathrm{BC}$-like layered compound, might increase its transition temperature to $\sim 50 \mathrm{~K}$. Theoretical work has also shown that single-walled carbon nanotubes (SWCNTs), as well as $\mathrm{BN}, \mathrm{B}_{\mathrm{x}} \mathrm{C}_{\mathrm{y}} \mathrm{N}_{\mathrm{z}}, \mathrm{BC}_{3}$ and hypothetical pure boron-based nanotubes, have comparable strain energies. ${ }^{15}$ This suggests that other stoichiometries of boron-containing carbon nanotubular structures may also be stable. Previous calculations that we have performed using the DFT tight binding (DFTTB) method have shown that charged $\mathrm{BC}$ nanotubes are stable and energetically viable. ${ }^{31}$

Due to the effects of electron confinement, small-radius carbon nanotubes have been predicted to possess very unusual features. These include a large split in the $G$-like raman modes, softening of the radial breathing modes, closing of the semiconducting gap, and possible enhancement of the electron-phonon coupling causing these ultranarrow nanotubes to be superconducting at relatively high temperatures $(\sim 15 \mathrm{~K}){ }^{32}$ It has also been shown that nanotubes of small radius can be relatively easily formed for some types of noncarbon nanotubes. For example, narrow $\mathrm{MoS}_{2}$ nanotubes with an almost uniform subnanometer diameter have been produced in a catalyzed reaction using $C_{60}$ as a growth promoter. ${ }^{33}$ In this paper we show that narrow $\mathrm{BC}$ nanotubes are energetically more stable than the corresponding strips obtained from boron carbide hexagonal sheets. We also show that doping $\mathrm{BC}$ tubes with $\mathrm{Cu}$ atoms results in the formation of covalent bonds between the dopant atoms and the carbon atoms on the tubular shell, while the bonding of $\mathrm{Li}$ with the boron and carbon atoms has a predominantly ionic character. These two effects are shown to dominate the structural and energetic properties of narrow $\mathrm{BC}$ tubes.

\section{METHOD AND PROCEDURE}

We have carried out $a b$ initio DFT spin-polarized calculations based on the plane wave pseudopotential method $(\mathrm{VASP})^{34,35}$ within the generalized gradient approximation $(\mathrm{GGA})^{36}$ for the exchange and correlation energy. For the modeling of the carbon, boron, and copper atomic interactions we have used standard ultrasoft (US) pseudopotentials, ${ }^{37}$ and for the Li atom we have employed the US pseudopotential with $p$-semi-core $\operatorname{states}^{37,38}$ and an energy cutoff of $286.7 \mathrm{eV}$.

Calculations have been conducted for: (i) an infinite, single layered $\mathrm{BC}$ hexagonal sheet with alternating boron and carbon atoms placed on the nodes of the "honeycomb" lattice, (ii) infinite, narrow boron-carbide strips which are "cut" from the BC hexagonal sheet (see Fig. 1), and (iii) infinite $\mathrm{BC}$ tubes formed from the infinite narrow boron carbide strips. The initial tubular geometries for the selected zigzag and armchair BC nanotubes were obtained by rolling up the corresponding infinite, narrow boron carbide sheets. 


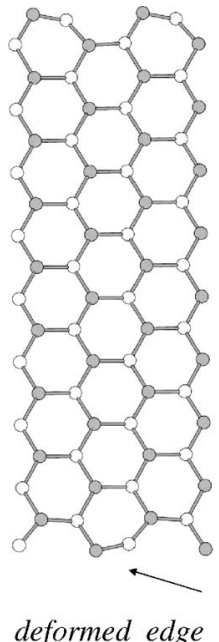

(a)

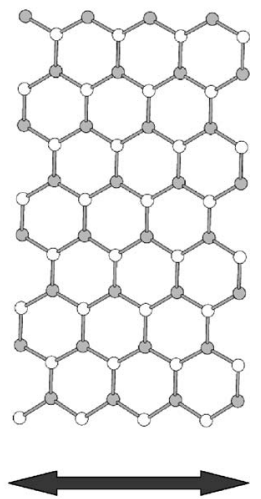

PBC
FIG. 1. Schematics of BC strips: (a) $(9,0)$, and (b) $(4,4)$. The direction of the periodic boundary conditions (PBC) is indicated by the large arrow. The boron atoms are denoted by white circles, and the carbon atoms by gray circles.

The dimensions of the unit cells have been chosen large enough to ensure that there are negligible interactions between neighboring strips or tubes.

The geometries of the boron carbide layers, strips, and tubes were fully relaxed with respect to the atomic positions and their unit cell lengths, using the conjugate gradient technique. The geometries were assumed to have converged when all of the forces acting on each of the atoms were less than $10^{-5} \mathrm{eV} / \AA$. The sets of $\mathbf{k}$ points were generated using the standard Monkhorst-Pack scheme. ${ }^{39}$ For optimization of the $\mathrm{BC}$ strips (which are infinite in the $x$ direction) we employed a $6 \times 2 \times 1$ k-point mesh, while for optimization of the infinite $\mathrm{BC}$ layer and tubes we have used a $3 \times 3 \times 3$ k-point mesh. Our tests have shown that the $3 \times 3 \times 3$ k-point mesh gives a very good representation of the structural properties of the tubes and the main energetic trends (i.e., the relative stabilities of the fully optimized doped and clean tubes) compared to finer k-point meshes. We have also conducted test calculations based on the two-ring model to check the stability of different sites for doping by Li atoms. ${ }^{40}$

The electronic structure for a single BC layer was calculated using a mesh of $24 \mathbf{k}$ points along the $\boldsymbol{\Gamma}-\mathbf{M}-\mathbf{K}-\boldsymbol{\Gamma}$ high symmetry directions of the first Brillouin zone. The electronic density of states (DOS) plot for the BC layer has been obtained using a smearing factor of $0.4 \mathrm{eV}$, while for the $\mathrm{BC}$ tubes a smearing factor of $0.2 \mathrm{eV}$ has been employed.

The energies of free $\mathrm{C}, \mathrm{B}, \mathrm{Li}$, and $\mathrm{Cu}$ atoms in vacuum, which are needed to calculate the binding energies for the $\mathrm{Li}(\mathrm{Cu})$ doped nanotubes and the heat of formation of the $\mathrm{BC}$ layer, were obtained using a large rectangular periodic unit cell with side length $\sim 30 \AA$ and the $3 \times 3 \times 3$ k-point mesh generated using the standard Monkhorst-Pack scheme. ${ }^{39}$

\section{RESULTS AND DISCUSSION}

\section{A. Infinite BC layer}

We have found that a single, infinite hexagonal BC layer is a planar structure. The converged total energy per boron
TABLE I. Heat of formation of a single infinite BC layer per B-C pair.

$\begin{array}{lr}\mathbf{E}_{\text {BClayer }}(\mathrm{eV} \text { per B-C pair) } & -15.021 \\ \mathbf{E}_{\text {Cvacuum }}(\mathrm{eV} \text { per B-C pair) } & -1.323 \\ \mathbf{E}_{\text {Bvacuum }}(\mathrm{eV} \text { per B-C pair) } & -0.298 \\ \text { Heat of formation of a BC layer } & 13.400 \\ \mathbf{E}_{\text {Bvacuum }}+\mathbf{E}_{\text {Cvacuum }}-\mathbf{E}_{\text {BClayer }} & \\ \text { (eV per B-C pair) }\end{array}$

carbon pair of the infinite hexagonal BC layer is much lower than the sum of the energies of the isolated carbon and boron atoms (see Table I). The BC layer is therefore energetically stable. The average bond length of the optimized layer was found to be $1.52 \AA$. The electronic band structure and the total DOS for this single, infinite BC layer are presented in Figs. 2(a) and 2(b), respectively. As can be seen, this system is metallic. The main features of the calculated electronic band structure are in good correspondence with the band structure previously calculated for a "charged" BC layer using the semiempirical DFT-TB method.

\section{B. Narrow BC strips}

To model narrow, infinite BC strips with open edges, we employed planar BC strips within a periodic orthorhombic
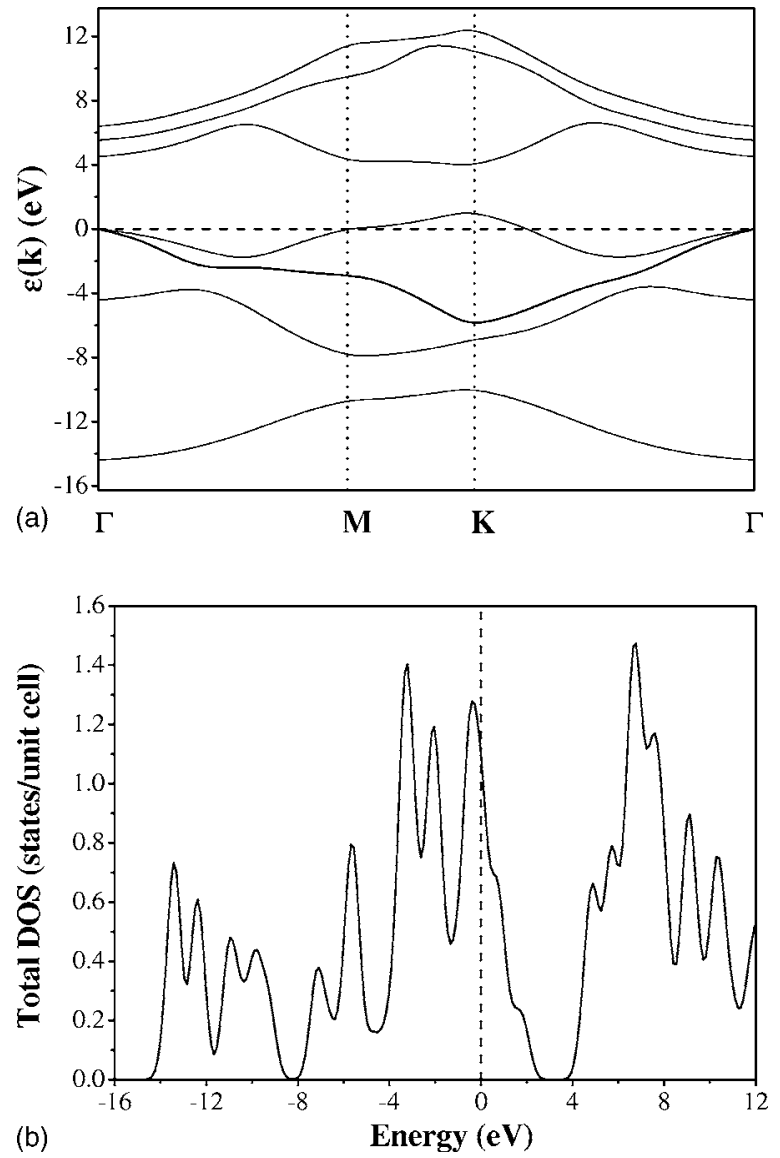

FIG. 2. Electronic structure for a single infinite BC layer: (a) band structure; (b) total DOS. The Fermi energy is indicated by the dashed line. 
TABLE II. Properties of clean BC nanotubes.

\begin{tabular}{lccc}
\hline \hline $\begin{array}{l}\text { BC nanotube } \\
(n, m)\end{array}$ & $\begin{array}{c}\text { Mean } \\
\text { diameter }(\AA)\end{array}$ & $\begin{array}{c}\text { Mean bond } \\
\text { length }(\AA)\end{array}$ & $\begin{array}{c}\text { Total energy } \\
(\mathrm{eV} / \text { atom })\end{array}$ \\
\hline$(6,0)$ & 4.99 & 1.54 & 7.38 \\
$(4,4)$ & 5.92 & 1.54 & 7.40 \\
$(5,5)$ & 7.45 & 1.53 & 7.43 \\
$(9,0)$ & 7.69 & 1.55 & 7.40 \\
$(10,0)$ & 8.46 & 1.55 & 7.41 \\
$(12,0)$ & 10.06 & 1.54 & 7.41 \\
\hline \hline
\end{tabular}

unit cell. This unit cell had a repeat length equivalent to two complete hexagonal rings along the direction corresponding to the longitudinal axis of the infinite tube that could be formed from one of these strips (see Fig. 1). In the other two orthogonal directions the cell repeat length was chosen large enough to prevent any interactions between the open edges of neighboring strips.

The structural stability of the infinite narrow strips was found to depend strongly on their width and edge topology. Moreover, the armchair edges of the zigzag strips underwent significantly more reconstruction during the course of optimization than the almost nondeformed open zigzag edges of the armchair strips [compare Figs. 1(a) and 1(b)].

\section{Clean BC nanotubes}

We have conducted geometry optimization calculations for clean $(6,0),(9,0),(10,0)$, and $(12,0)$ zigzag BC tubes, and $(4,4)$ and $(5,5)$ armchair BC tubes. Values for some of the properties of these tubes obtained from these calculations are presented in Table II. The mean BC bond length for all calculated tubes was found to be $1.54 \AA$, which is slightly longer than the value of $1.52 \AA$ determined for the hexagonal layer. The $(6,0)$ tube, which is the narrowest we have studied [see Fig. 3(a)], was found to have a mean diameter of $4.99 \AA$, while our largest calculated BC tube $[(12,0)]$ has a mean diameter of $10.06 \AA$. All the $\mathrm{BC}$ tubes were found to preserve both their hollow shape and the hexagonal boron carbon network on the tubular shells. The tubular cross section of the narrow armchair $(4,4) \mathrm{BC}$ tube was found to acquire a symmetric rectangular shape, as can be seen from Fig. 3(b). Figure 4 shows the total energy of clean BC tubes, and the corresponding $\mathrm{BC}$ strips, plotted as a function of the initial diameter of the tubes that are formed from the strips. These results show that the formation of narrow BC nanotubes is energetically more favorable than the formation of the corresponding BC strips. This is due to the elimination of the chemical energy of the dangling bonds at the open edges of the strips when the tubular structures are formed. We have also found that the total energy of clean BC nanotubes increases with decreasing radius, and hence with increasing curvature of the tubes (see Fig. 4). This energy dependence is similar to that found for SWCNTs (Ref. 41).

In the remainder of this paper we shall only consider the most narrow of the above-mentioned tubes; namely, the $(6,0)$ zigzag, and $(4,4)$ armchair BC tubes. The calculated total

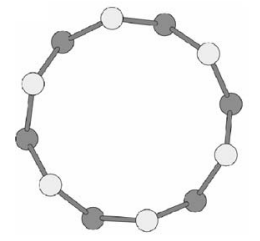

(a)

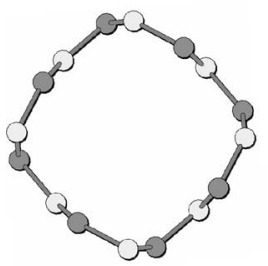

(b)
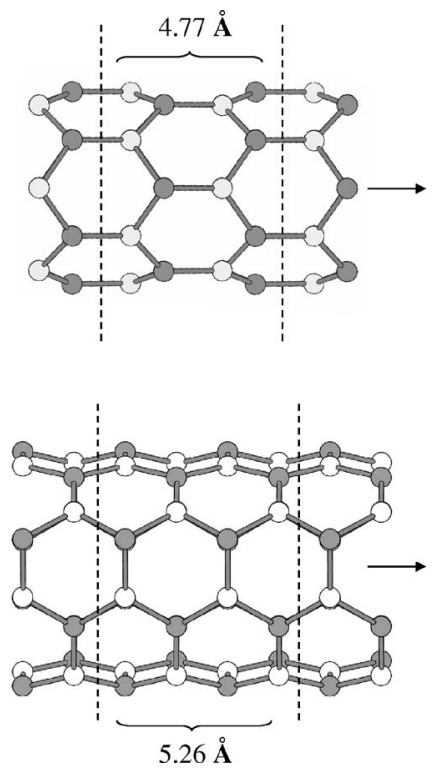

FIG. 3. End and side views of two optimized small diameter BC nanotubes: (a) $(6,0)$, and (b) $(4,4)$. The dotted lines indicate the repeat length of the periodic unit cell.

DOS for these two BC nanotubes are shown in Fig. 5(a). Both of these tubes were found to be metallic. A charge density plot for the clean $(4,4)$ BC tube is presented in Fig. 5(b) and shows pronounced charge asymmetry in the boron carbon bonding with greater charge localization in the vicinity of the carbon atoms. Similar charge distributions within the boron carbon hexagonal network have been shown to occur for both single-walled $\mathrm{BC}_{3}$ tubes ${ }^{42}$ and $\mathrm{LiBC}$ clusters. $^{43}$

\section{Doped BC tubes}

It has been predicted that doping of small-diameter carbon nanotubes may enhance their superconducting properties. ${ }^{32}$ One of the aims of the present paper, inspired by these ideas, was therefore to determine the extent to which doping by $\mathrm{Li}$ or $\mathrm{Cu}$ atoms could modify the structural, energetic, and electronic properties of small-diameter BC nanotubes. Test calculations suggested that positioning the chemisorbed metal

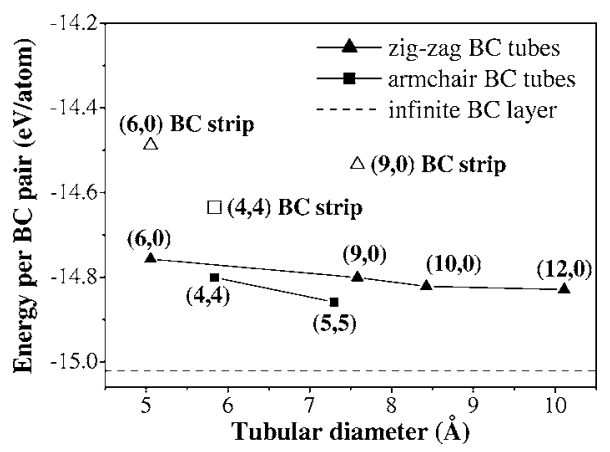

FIG. 4. Total energies (in eV per BC pair) of BC tubes and strips as a function of their corresponding tubular diameter (taken to be that of the unrelaxed tube). The value for the infinite $\mathrm{BC}$ layer is indicated by the dashed line. 


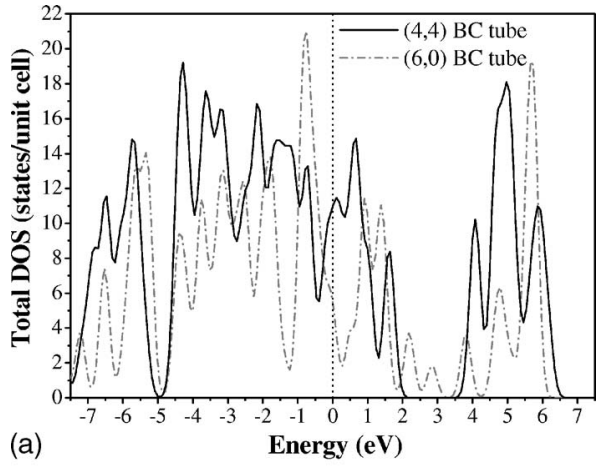

(a)

Energy (eV)

(b)

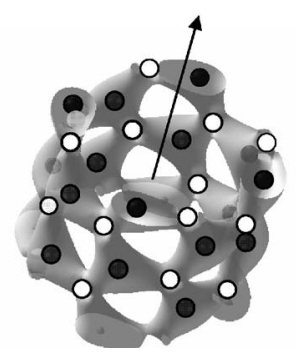

FIG. 5. (a) Total DOS for the $(6,0)$ zigzag and $(4,4)$ armchair $\mathrm{BC}$ nanotubes, and (b) charge density isosurface plotted for a section of the clean $(4,4) \mathrm{BC}$ tube. The arrow indicates the longitudinal axis of the tube. The boron atoms are denoted by the white circles and the carbon atoms by the dark circles.

atoms inside an isolated BC tube is more energetically favorable than placing them at sites on the outer surface of the tube. We have found that doping by metal atoms at sites outside isolated tubes leads to corrugation of the tubes, and produces less stable structures than inside doping of the tubes, with the energy difference per cell between the most stable inside and outside sites for the $(4,4) \mathrm{BC}$ tube being $1.55 \mathrm{eV}$ for a $\mathrm{Cu}$ atom, and $0.88 \mathrm{eV}$ for a $\mathrm{Li}$ atom per cell. Our test calculations on the doping of flat BC layers with $\mathrm{Li}(\mathrm{Cu})$ atoms indicated that the dopant atom prefers to stay at a distance of $\sim 1.69$ (1.92) $\AA$ from the BC hexagonal layer, rather than being embedded in the surface of the layer. Hence, we have focused our attention in this paper on studying the inside doping of $\mathrm{BC}$ tubes. Doping of these tubes was achieved by placing $\mathrm{Li}$ or $\mathrm{Cu}$ atoms at different adsorption sites inside $\mathrm{BC}$ nanotubes. For the $(6,0)$ zigzag $\mathrm{BC}$ tubes the doping concentration was $1 \mathrm{Cu}$ atom, or 1-3 $\mathrm{Li}$ atoms, per 12 boron carbon pairs in the periodic unit cell, while for the $(4,4)$ armchair $\mathrm{BC}$ tube we used $1 \mathrm{Li}$ atom, or $1-2 \mathrm{Cu}$ atoms, per 16 boron carbon pairs.

\section{E. BC tubes doped with $\mathrm{Li}$}

Doping the interior of narrow BC tubes with $\mathrm{Li}$ was found to significantly affect the structural properties of the tubes. The mean BC bond length for both the Li doped $(6,0)$ and $(4,4) \mathrm{BC}$ tubes was found to be $1.55 \AA$, which is slightly longer than the value of $1.54 \AA$ determined for the corresponding clean BC tubes (see Table II). As shown in Fig. 6 and Table III, the effects of doping were found to depend on both the helicity and diameter of the tubes. The $(6,0) \mathrm{BC}$
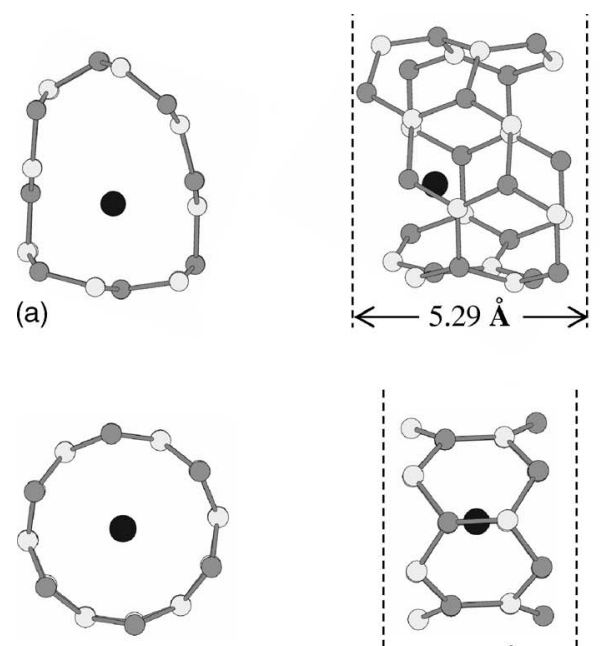

(b)
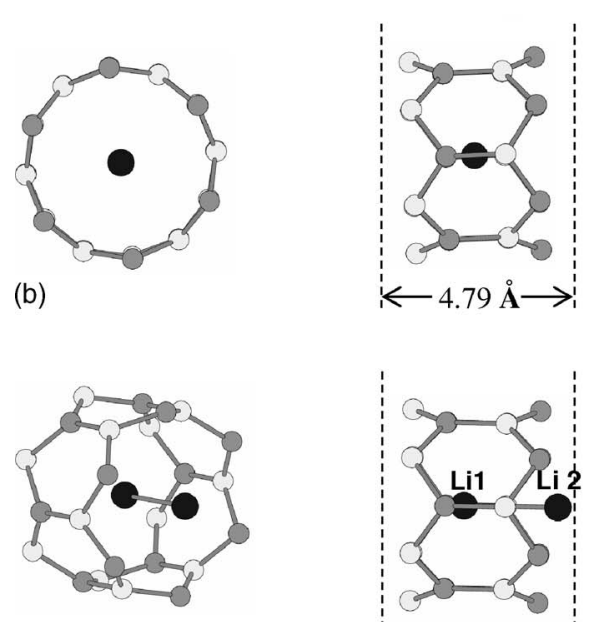

(c)
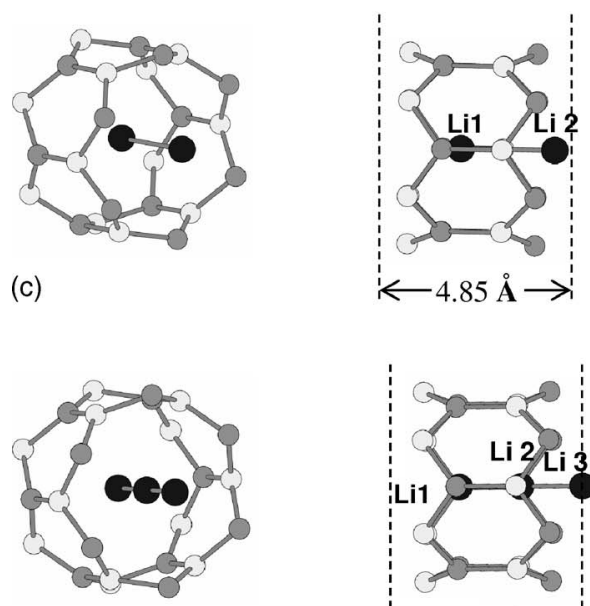

(d)

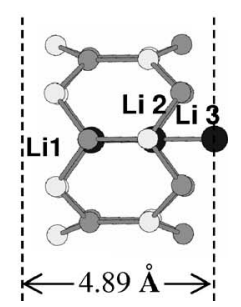

FIG. 6. Optimized structures of Li-doped BC nanotubes: (a) $(4,4)$ doped with one $\mathrm{Li},(\mathrm{b})(6,0)$ doped with one $\mathrm{Li},(\mathrm{c})(6,0)$ doped with two $\mathrm{Li}$ atoms, and (d) $(6,0)$ doped with three $\mathrm{Li}$ atoms. The atoms are denoted by white (B), gray $(\mathrm{C})$, and black (Li) circles. The dashed vertical lines indicate the repeat length of the periodic unit cell.

nanotube with one $\mathrm{Li}$ atom per cell was found to retain a cylindrical shape with the $\mathrm{Li}$ atom positioned on the central axis of the tube, as shown in Fig. 6(b). By contrast, in the most stable structure for the $(4,4)$ Li-doped BC nanotube with one $\mathrm{Li}$ atom per cell, the $\mathrm{Li}$ atom is positioned away from the central axis of the tube, as shown in Fig. 6(a), and prefers to occupy a position which is closer to one of the carbon atoms on the tubular shell (C-Li distance $\sim 2.28 \AA$ ) than to the boron atoms. While the hexagonal BC network of the $(4,4)$ tube is retained, its shape is no longer symmetrical. The observed dependence of the $\mathrm{Li}$ atom position on the size of the $\mathrm{BC}$ tube is similar to results obtained from modeling the insertion of $\mathrm{Li}$ into carbon nanotubes ${ }^{40,44}$ where it was shown that the most stable position for a $\mathrm{Li}$ atom inside a SWCNT is strongly dependent on its radius.

In order to model the most energetically favorable positions for a $\mathrm{Li}$ atom inside a $\mathrm{BC}$ nanotube we have employed a simple "two-ring model" (shown in Fig. 7). This model 
TABLE III. Properties of isolated small-diameter BC tubes doped inside with Li atoms. All the distances are in $\AA$.

\begin{tabular}{lcccc}
\hline \hline $\begin{array}{l}\text { Structure, } n \text { Li atoms } \\
\text { Inside }(n, m) \text { BC tube }\end{array}$ & $\begin{array}{c}1 \mathrm{Li} \\
(4,4)\end{array}$ & $\begin{array}{c}1 \mathrm{Li} \\
(6,0)\end{array}$ & $\begin{array}{c}2 \mathrm{Li} \\
(6,0)\end{array}$ & $\begin{array}{c}3 \mathrm{Li} \\
(6,0)\end{array}$ \\
\hline Mean B-C bond length & 1.55 & 1.55 & 1.55 & 1.56 \\
Shortest B-C bond length & 1.50 & 1.54 & 1.55 & 1.54 \\
Longest B-C bond length & 1.61 & 1.55 & 1.56 & 1.58 \\
Tubular cross section (max) & 6.05 & 5.02 & 4.96 & 5.00 \\
Tubular cross section (min) & 4.48 & 4.97 & 4.94 & 4.86 \\
C-Li nearest neighbor distance & 2.28 & 2.63 & 2.53 & 2.56 \\
B-Li nearest neighbor distance & 2.67 & 2.61 & 2.69 & 2.57 \\
Li1-Li2 distance & - & - & 2.43 & 1.64 \\
Li2-Li3 distance & - & - & - & 1.62 \\
Average binding energy (eV) & 2.85 & 3.79 & 3.21 & 1.60 \\
Heat of formation $(\mathrm{eV})$ & 2.85 & 3.79 & 2.63 & -1.62 \\
\hline \hline
\end{tabular}

was used by Kar et al. ${ }^{40}$ to study the interaction of inserted $\mathrm{Li}$ atoms with the walls of SWCNTs. In this model, the opposite walls of the nanotube are represented by two hexagonal benzenelike rings with boron and carbon atoms placed alternately. One-point energy calculations were carried out for different distances between the rings (which corresponded to different diameters of the BC tubes), and for the $\mathrm{Li}$ atom occupying different positions between the rings. The minimal energy distance of the $\mathrm{Li}$ atom from one of the hexagonal rings was found to be in the range $1.5 \AA-2.4 \AA$,

(a)
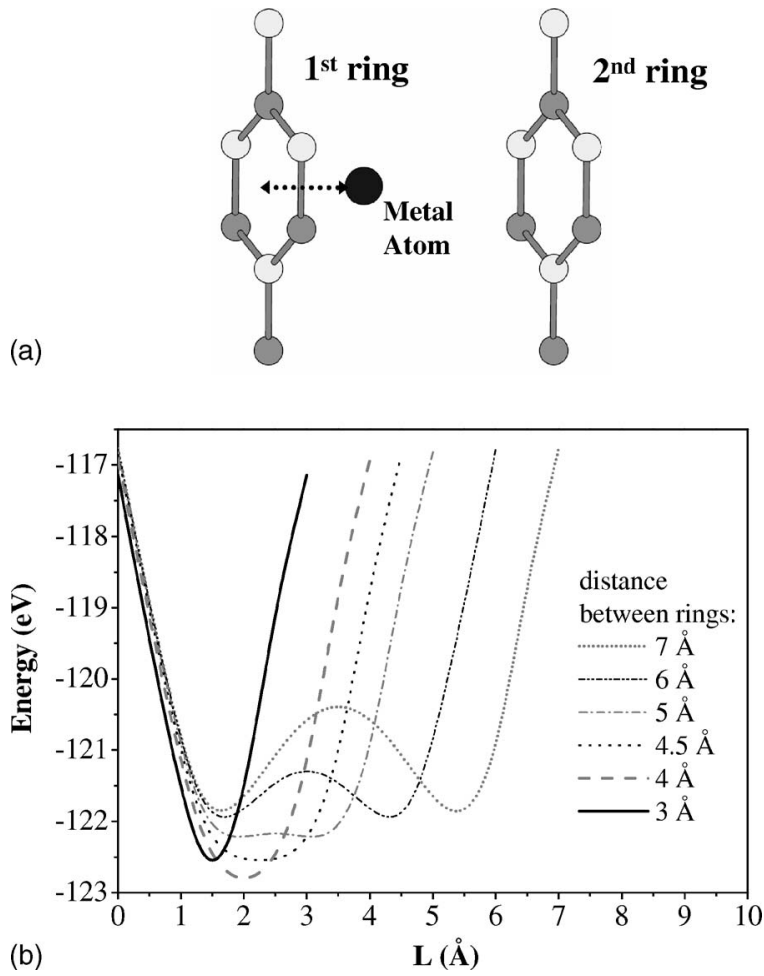

FIG. 7. (a) Two-ring model where $\mathrm{L}$ is the distance between the metal atom and the first BC ring. (b) Variation of the total energy as the $\mathrm{Li}$ atom moves between the two $\mathrm{BC}$ rings for different distances between the rings. depending on the separation between the hexagonal rings [see Fig. 7(b)]. When the distance between the two hexagonal rings is in the range $3 \AA-4.5 \AA$, which would correspond to very narrow tubes, our calculations show that the most stable equilibrium position for the $\mathrm{Li}$ atom is located at the central position between the rings. This corresponds to the geometrical center of the modeled tubes. For larger ring separations, the central position between the two rings will not be a minimum energy position for a $\mathrm{Li}$ atom inserted into the tube. These simple calculations can partly explain the difference in the effects of doping by $\mathrm{Li}$ on the geometric structure of the more narrow $(6,0) \mathrm{BC}$ tube compared to the wider $(4,4)$ tube.

We have also analyzed the energetics of doping narrow $\mathrm{BC}$ tubes with metal atoms. The average binding energy for doping a BC tube with $N$ metal atoms has been calculated using the formula (Refs. 45 and 46)

$$
\begin{aligned}
E_{\mathrm{BIND}}= & {\left[E_{\mathrm{TOT}}(\text { BCtube })+N \times E_{\mathrm{TOT}}(M \text { free })\right.} \\
& \left.-E_{\mathrm{TOT}}\left(\text { BCtube } \sim M_{\mathrm{N}}\right)\right] / N,
\end{aligned}
$$

where $E_{\mathrm{TOT}}\left(\mathrm{BCtube} \sim M_{\mathrm{N}}\right)$ is the total energy of the fully optimized $\mathrm{BC}$ nanotube doped with $N$ metal atoms, $E_{\mathrm{TOT}}(\mathrm{BCtube})$ is the total energy of the optimized clean $\mathrm{BC}$ nanotube, and $E_{\mathrm{TOT}}(M$ free $)$ is the energy of the dopant metal atom in vacuum. The calculated total energy for an isolated lithium atom was $-0.2825 \mathrm{eV}$. We found that the binding energy corresponding to a $\mathrm{BC}$ tube with one interior $\mathrm{Li}$ atom per cell was greater in the case of the smaller diameter $(6,0)$ $\mathrm{BC}$ tube $(3.79 \mathrm{eV})$ than for the $(4,4) \mathrm{BC}$ tube $(2.85 \mathrm{eV})$. This difference in the calculated binding energies may be related to the different number of atoms on the boron carbon tubular shells of the (cylindrical) zigzag $(6,0)$ and (deformed) armchair $(4,4)$ tubes which interact strongly with the Li atom.

Previous studies of doping SWCNTs with Li have shown an almost complete charge transfer from the Li atoms to the carbon shell. ${ }^{47}$ We might thus expect that charge transfer from Li to the shell of a BC nanotube would also be significant due to the larger electronegativity of the boron and carbon atoms compared with Li. In fact, similar conclusions 

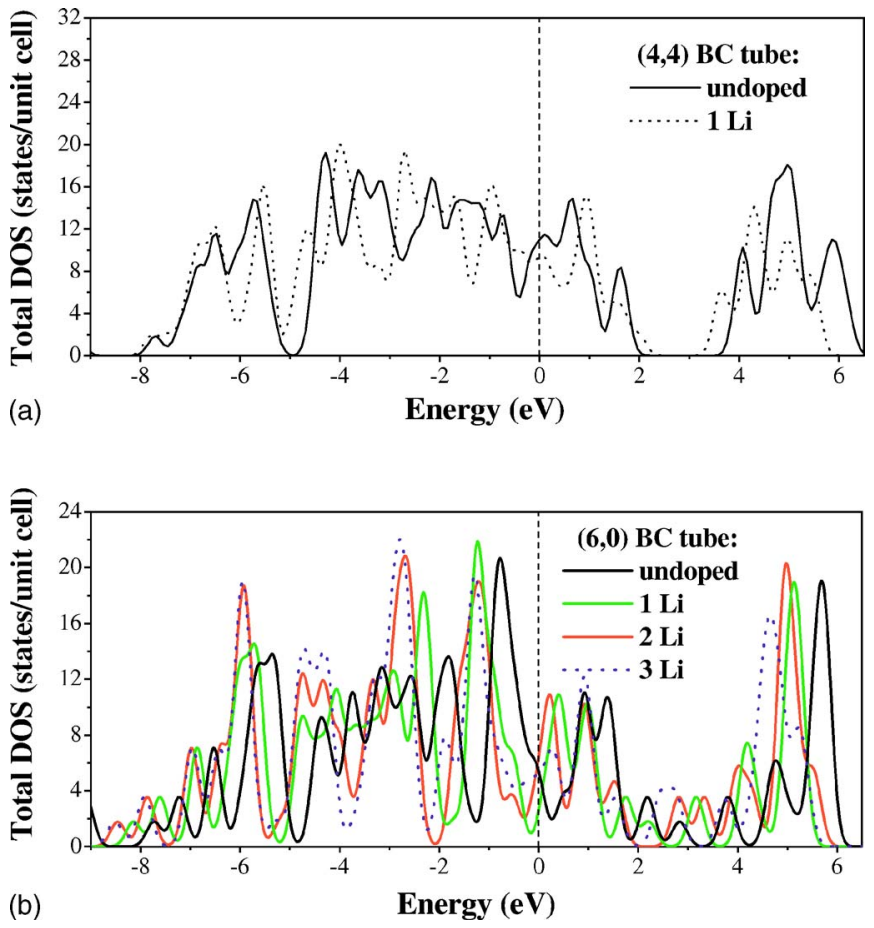

FIG. 8. (Color online) (a) Total DOS for $(4,4)$ BC tubes both clean and doped with one lithium atom, and (b) total DOS for $(6,0)$ $\mathrm{BC}$ tubes both clean and doped with 1-3 $\mathrm{Li}$ atoms.

have been made by Zhou et al. who theoretically studied lithium adsorption in boron-doped SWCNTs. (Ref. 48). To confirm that the interaction of $\mathrm{Li}$ with a $\mathrm{BC}$ nanotube has a predominantly ionic character, we have calculated the charge density difference between a $(6,0)$ BC tube doped with 1 lithium atom and a clean $(6,0) \mathrm{BC}$ tube. We then integrated this charge difference within a sphere of radius $1.0 \AA$ (which is $50 \%$ larger than the ionic radius of $\mathrm{Li}$ ) centered on the $\mathrm{Li}$ atom. The total charge within this sphere was found to be only 0.076 units of electron charge. We can thus confidently conclude that the Li atom gives up most of its charge to the $\mathrm{BC}$ shell.

The DOS structures for the $(4,4)$ and $(6,0)$ BC nanotubes doped with one Li are shown in Figs. 8(a) and 8(b). As can be seen, these Li-doped BC tubes are metallic. We also observe that, apart from a shift in the Fermi energy, doping with one $\mathrm{Li}$ atom per cell only produces small changes in the valence band DOS of the $(6,0) \mathrm{BC}$ tubes. This is evidenced by the very similar structure of the DOS for the clean and Li-doped $(6,0)$ BC tubes [see Fig. 8(b)]. In the case of the $(4,4)$ BC tubes, however, where doping produces significant structural changes, the DOS of the clean and Li-doped tubes are seen to be fairly different.

\section{F. Doping the $(6,0) \mathrm{BC}$ tube with 2 and $3 \mathrm{Li}$ atoms}

As we have shown, the $(6,0) \mathrm{BC}$ tube preserves its shape upon doping with one $\mathrm{Li}$ atom per cell. We have thus conducted a series of spin-polarized calculations to study the effects of increased concentration of $\mathrm{Li}$ on the structural and electronic properties of $(6,0) \mathrm{BC}$ tubes. As shown in Figs. 6(c) and 6(d), the optimized $(6,0)$ BC tube doped with two or three $\mathrm{Li}$ atoms per cell retains a cylindrical shape with the $\mathrm{Li}$ atoms positioned along the central axis of the tube forming a one-dimensional wire. The structural details of these tubes do not differ significantly from those of a $(6,0) \mathrm{BC}$ tube doped with just one $\mathrm{Li}$ atom, as can be seen from comparing the datasets in Table III. The periodic unit cell, whose length was allowed to vary along the longitudinal axis of the tube, was found to undergo minimal stretching to accommodate the additional $\mathrm{Li}$ atoms inside the tube. The diameters of the $(6,0) \mathrm{BC}$ tubes doped with two or three Li atoms are slightly smaller than the diameters of both the clean $(6,0) \mathrm{BC}$ tube and that doped with one $\mathrm{Li}$ atom. The $\mathrm{Li}-\mathrm{Li}$ distance for a tube doped with two $\mathrm{Li}$ atoms is $2.43 \AA$, and reduces to $\sim 1.63 \AA$ for a tube doped with three Li atoms (see Table III).

The average binding energy values for the $(6,0) \mathrm{BC}$ tubes doped with two and three Lithium atoms are also presented in Table III. The average binding energy for the $(6,0) \mathrm{BC}$ tube doped with three Li atoms is observed to be much lower than the other values. This is most likely due to increased repulsion of the lithium atoms as the distance between them is decreased. Another important parameter to characterize doping is the heat of formation, $H_{\mathrm{N}}$. This can be calculated using the formula (Refs. 49 and 50)

$$
\begin{aligned}
H_{\mathrm{N}}= & E_{\mathrm{TOT}}\left(\text { BCtube } \sim M_{\mathrm{N}-1}\right)+E_{\mathrm{TOT}}(M \text { free }) \\
& -E_{\mathrm{TOT}}\left(\text { BCtube } \sim M_{\mathrm{N}}\right),
\end{aligned}
$$

where $E_{\mathrm{TOT}}\left(\mathrm{BCtube} \sim M_{\mathrm{N}}\right)$ is the total energy of the fully optimized nanotube doped with $N$ metal atoms, $E_{\mathrm{TOT}}\left(\mathrm{BCtube} \sim M_{\mathrm{N}-1}\right)$ is the total energy of a fully optimized nanotube doped with $(N-1)$ metal atoms, and $E_{\mathrm{TOT}}(M$ free $)$ is the energy of a single dopant atom in vacuum. The calculated values for the heat of formation are presented in Table III. The data shows that the heat of formation decreases with increasing doping. The negative value of the heat of formation for three dopant atoms per unit cell indicates that it would be difficult to achieve high concentrations of lithium by "inside" doping of an isolated $(6,0) \mathrm{BC}$ tube.

The DOS plots corresponding to $(6,0) \mathrm{BC}$ tubes doped with two and three Li atoms are also presented in Fig. 8(b). The peak structure of the total DOS for the $(6,0)$ BC tubes doped with one, two, and three $\mathrm{Li}$ atoms only changes slightly from that of the clean $(6,0) \mathrm{BC}$ tube. Increasing the concentration of $\mathrm{Li}$ atoms in the $(6,0) \mathrm{BC}$ tube shifts the Fermi energy upwards, but does not lead to any significant change in the density of states at this level. The charge density plot for the $(6,0) \mathrm{BC}$ tube doped with three $\mathrm{Li}$ atoms presented in Fig. 9 shows that the electronic charge is mostly located on the $\mathrm{BC}$ hexagonal network, with only a small amount of charge spread along the line joining the $\mathrm{Li}$ atoms. This shows that there is an absence of covalent bonding between the $\mathrm{Li}$ atoms and the atoms on the tubular shell.

\section{G. BC tubes doped by $\mathrm{Cu}$}

Doping with one $\mathrm{Cu}$ atom per cell drastically changes the shape of the tubular shell of the $(6,0)$ zigzag BC tube to that of an ellipse [see Fig. 10(a)]. The minimum energy configu- 


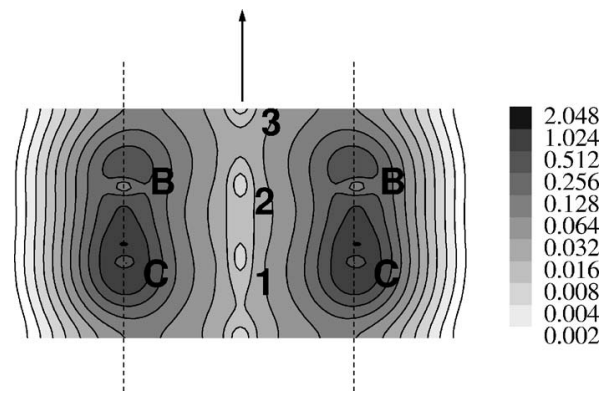

FIG. 9. Charge density contour plot for the $(6,0) \mathrm{BC}$ tube doped with three lithium atoms for a plane containing the longitudinal axis of the tube. The vertical dashed lines indicate the walls of the tube. The contour values are in units of electron charge.

ration for the $(4,4)$ armchair $\mathrm{BC}$ tube is a rectangularlike structure with the $\mathrm{Cu}$ atom positioned on the central axis of the deformed shell [Fig. 10(b)]. The BC bond length for the $\mathrm{Cu}$-doped $(4,4) \mathrm{BC}$ tube was found to range from 1.46 to $1.62 \AA$, with an average value of around $1.53 \AA$ (see Table IV). For the $\mathrm{Cu}$-doped $(6,0) \mathrm{BC}$ tube, the corresponding bond length values ranged from 1.50 to $1.60 \AA$ with an average bond length of $1.55 \AA$. After geometry optimization, the interatomic distance between the $\mathrm{Cu}$ atoms of neighboring cells was $4.84 \AA$ for the $(6,0)$ tube, and $5.29 \AA$ for the $(4,4)$ tube. For such interatomic separations, interaction between neighboring $\mathrm{Cu}$ atoms should be negligible.

The differences in structure of the small-radius $\mathrm{Cu}$-doped and Li-doped BC tubes can be explained by the different properties of the dopant atoms, such as their size and bonding preferences. $\mathrm{Li}$ atoms, which are quite small and very light, have only one $s$ electron in their outer shell and hence tend to form ionic bonds by giving up this electron to more electronegative atoms (boron and carbon atoms in our case). $\mathrm{A} \mathrm{Cu}$ atom, on the other hand, is much larger and heavier, and due to its electronic structure can form either ionic ( $4 \mathrm{~s}$ electrons) or covalent ( $3 d$ electrons) bonds, depending on the environment. The tendency of $\mathrm{Cu}$ atoms to form a covalent bond, and the different shell symmetry of the $(6,0)$ zigzag and $(4,4)$ armchair BC tubes, can explain the observed differences in the atomic structures of the $\mathrm{Cu}$-doped tubes. The unique combination of the value of the diameter and the symmetry of the armchair $(4,4) \mathrm{BC}$ tube makes strong inter- (a)
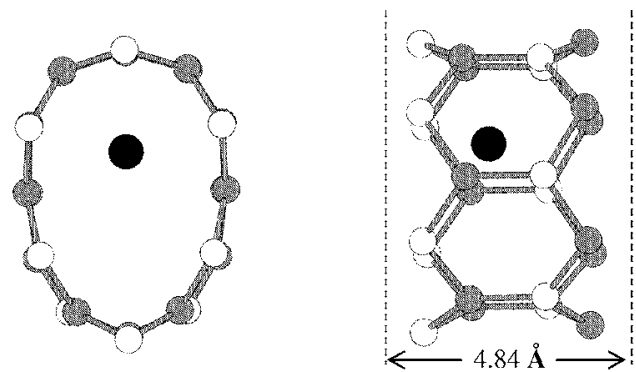

(b)
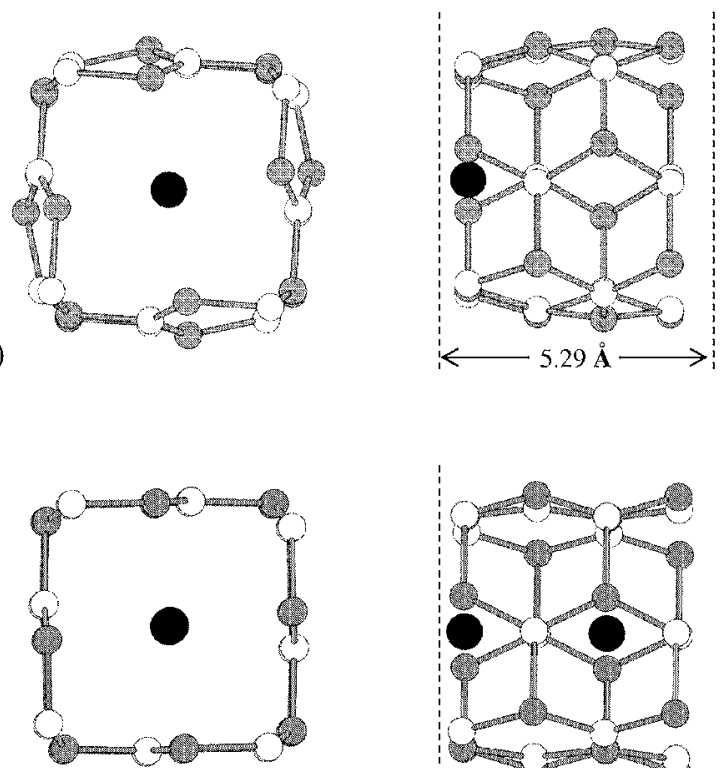

(c)

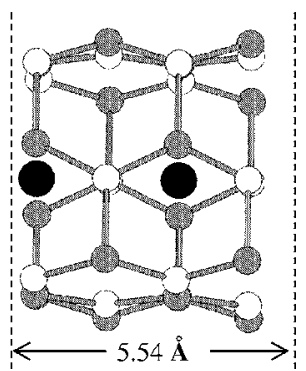

FIG. 10. Optimized structures of Cu-doped BC nanotubes: (a) $(6,0) \mathrm{BC}$ tube doped with one $\mathrm{Cu}$ atom, (b) $(4,4) \mathrm{BC}$ tube doped with one $\mathrm{Cu}$ atom, and (c) $(4,4) \mathrm{BC}$ tube doped with two $\mathrm{Cu}$ atoms. The $\mathrm{Cu}$ atoms are denoted by the black circles and the vertical dashed lines indicate the extent of the periodic unit cell.

actions between the $d$ orbitals of the dopant $\mathrm{Cu}$ atom(s) and four Carbon atoms on opposite sides of the tubular shell especially favorable, and results in the stabilization of the rectangularlike shape of the doped $(4,4)$ tube shown in Fig. 10(b). This is further illustrated by the charge density plot presented in Fig. 11(b). The smaller diameter zigzag $(6,0)$

TABLE IV. Properties of the $(6,0)$ and $(4,4)$ BC nanotubes doped with $\mathrm{Cu}$. All the distances are in $\AA$.

\begin{tabular}{lccc}
\hline \hline Cu-doped BC nanotube & 1 Cu-doped $(6,0)$ & 1 Cu-doped $(4,4)$ & 2 Cu-doped $(4,4)$ \\
\hline Mean B-C bond length & 1.55 & 1.53 & 1.55 \\
Shortest B-C bond length & 1.50 & 1.46 & 1.50 \\
Longest B-C bond length & 1.60 & 1.62 & 1.61 \\
Mean B-Cu distance & 2.37 & 3.01 & 2.79 \\
Mean C-Cu bond length & 2.15 & 2.20 & 2.36 \\
Tubular cross section (max) & 5.74 & 6.18 & 5.98 \\
Tubular cross section (min) & 3.95 & 4.40 & 4.73 \\
Binding energy (eV) & 4.32 & 2.98 & 3.37 \\
Heat of formation (eV) & & & 3.76 \\
\hline \hline
\end{tabular}


(a)
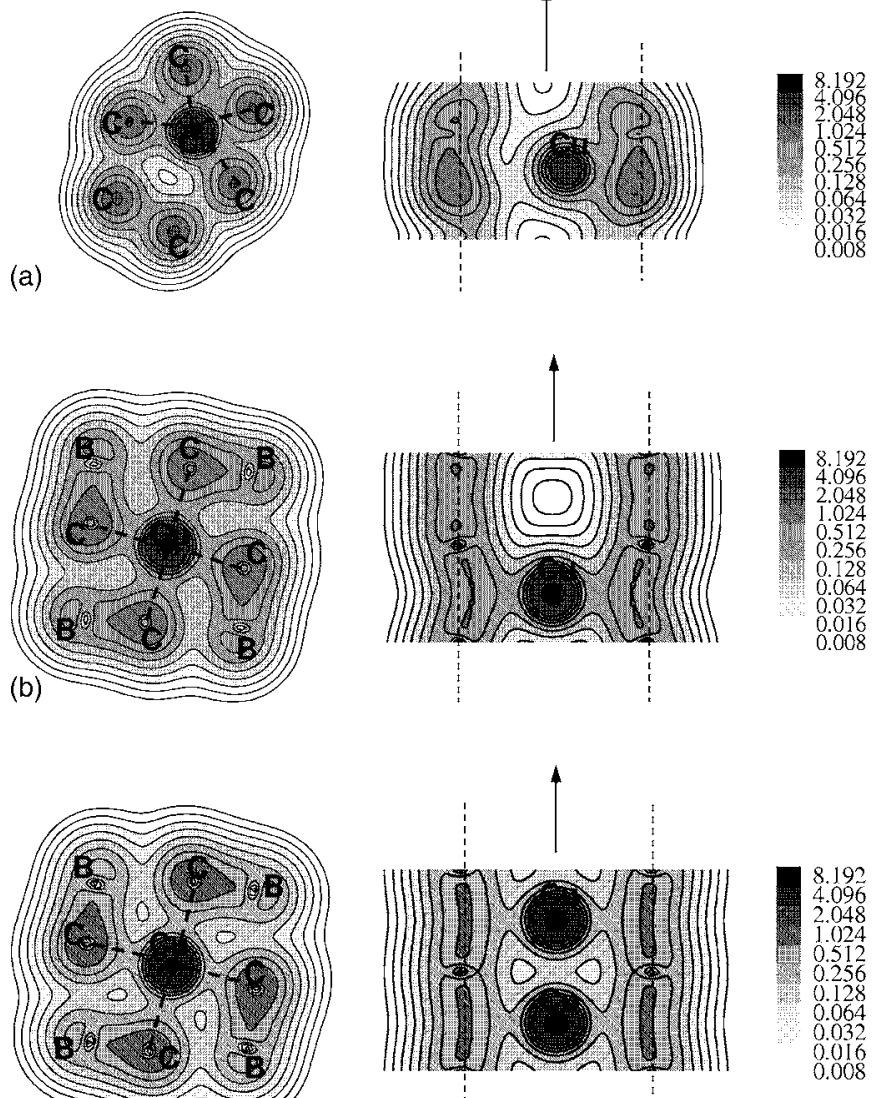

(c)
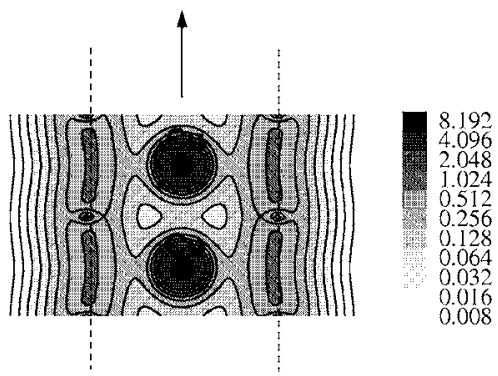

FIG. 11. Slices of the total charge density distribution for $\mathrm{Cu}-$ doped BC tubes: (a) $(6,0) \mathrm{BC}$ tube with one $\mathrm{Cu}$ atom per cell, (b) $(4,4) \mathrm{BC}$ tube with one $\mathrm{Cu}$ atom per cell, and (c) $(4,4) \mathrm{BC}$ tube with two $\mathrm{Cu}$ atoms per cell. In each case, the cross section on the left is for a plane containing the copper atom(s) and normal to the longitudinal axis of the tube, while the plot on the right includes the $\mathrm{Cu}$ atom(s) and the longitudinal axis of the tube. The graduated scales on the extreme right define the charge distribution in units of electron charge. The arrows denote the tube axis, and the vertical dashed lines indicate the walls of the tubes. The gray dashed lines in the left-hand figures indicate the directions of the nearest neighbor interactions between the dopant $\mathrm{Cu}$ atom(s) and the carbon atoms on the tubular shell.

tube, on the other hand, does not provide such commensurate symmetric sites for bonding of the $\mathrm{Cu}$ atom with the carbon atoms on the shell. This results in an elliptical deformation of the cross section of the $(6,0)$ BC tube upon doping, as shown in Figs. 10(a) and 11(a).

We have also studied the effect of increased concentration of $\mathrm{Cu}$ atoms on the properties of the $(4,4) \mathrm{BC}$ tube. The optimized structure of the $(4,4) \mathrm{BC}$ tube doped with two $\mathrm{Cu}$ atoms per cell is shown in Fig. 10(c). As can be seen, this tube retains the rectangularlike cross section produced by doping with just one $\mathrm{Cu}$ atom per cell. Also, as in the case of doping with one $\mathrm{Cu}$ atom, the two $\mathrm{Cu}$ atoms form predominantly covalent bonds with the four closest carbon atoms of the tubular shell. The distance separating the two $\mathrm{Cu}$ atoms in this case is only $2.77 \AA$, which gives rise to significant interactions between them, as illustrated in Fig 11(c).
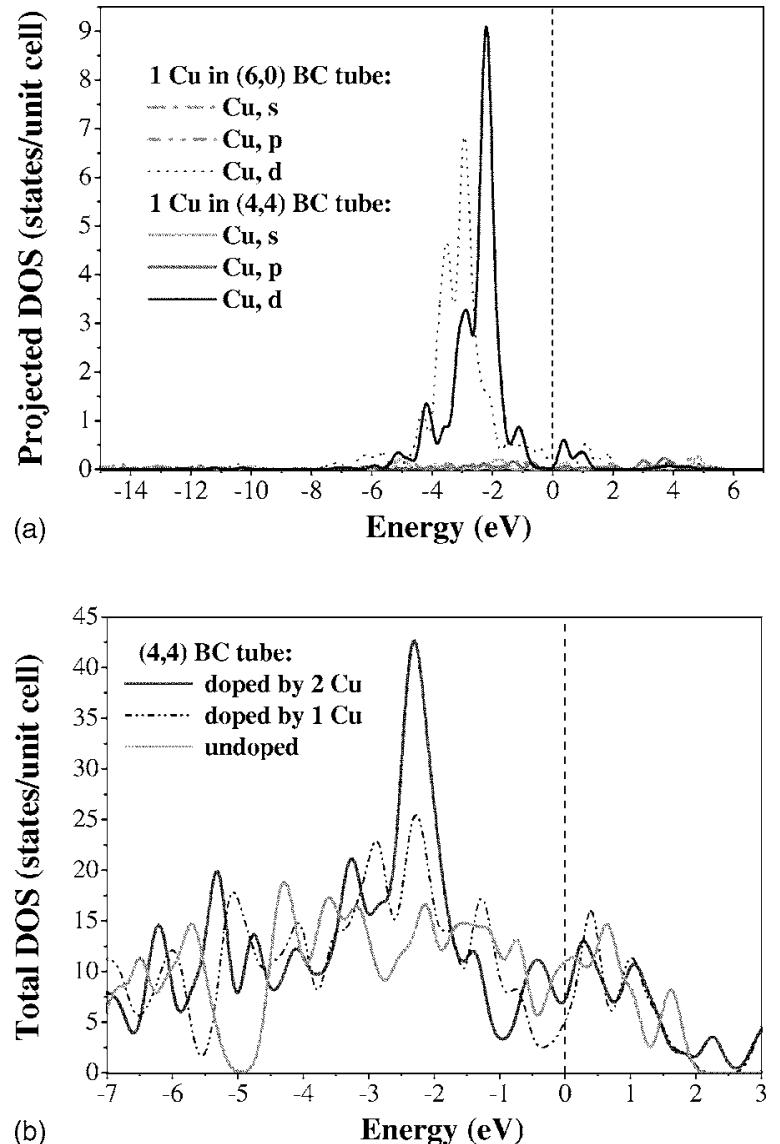

FIG. 12. DOS for the $(6,0)$ and $(4,4) \mathrm{Cu}$-doped BC tubes: (a) $l$-projected density of states for the copper atom for doping with just one $\mathrm{Cu}$ atom per cell, (b) total DOS for a clean $(4,4) \mathrm{BC}$ tube, and $(4,4) \mathrm{BC}$ tubes doped with one and two $\mathrm{Cu}$ atoms per cell. The Fermi energy in each case is indicated by the vertical dashed line.

The average binding energy and heat of formation for the $\mathrm{Cu}$ doped $(4,4) \mathrm{BC}$ tubes have been calculated using expressions (1) and (2) with $M=\mathrm{Cu}$, and a total energy for a free copper atom of $-0.234 \mathrm{eV}$. The results are presented in Table IV. The average binding energy of $4.32 \mathrm{eV}$ for a $(6,0) \mathrm{BC}$ tube doped with one $\mathrm{Cu}$ atom per cell is significantly higher than that for the corresponding $(4,4) \mathrm{BC}$ tube $(2.98 \mathrm{eV})$. Doping the $(4,4) \mathrm{BC}$ tube with a second $\mathrm{Cu}$ atom is seen to be energetically favorable with the average binding energy increasing by $0.39 \mathrm{eV}$. The corresponding heat of formation is positive and equal to $3.76 \mathrm{eV}$. This is related to the formation of $\mathrm{Cu}-\mathrm{Cu}$ bonding as evidenced by the charge density distribution in Fig. 11(c).

The DOS of the $(6,0)$ and $(4,4) \mathrm{BC}$ tubes doped with $\mathrm{Cu}$ atoms are shown in Fig. 12. In each case, the $\mathrm{Cu}$-doped $\mathrm{BC}$ tubes are seen to be metallic. Comparison with the DOS of the corresponding clean BC tubes plotted in Fig. 5(a) reveals that doping with $\mathrm{Cu}$ produces significant changes. The valence band DOS of these narrow BC tubes is strongly influenced by the formation of covalent bonds between the copper atom(s) and the boron carbide tubular shell. For both the $(6,0)$ and $(4,4) \mathrm{BC}$ tubes with a single $\mathrm{Cu}$ atom per cell, the DOS in the valence band region is dominated by a very large contribution from the $\mathrm{Cu}-d$ states [Fig. 12(a)]. Increasing the 
doping of the $(4,4) \mathrm{BC}$ tube by adding a second $\mathrm{Cu}$ atom per cell also significantly changes the DOS structure. This can be seen from Fig. 12(b). As for the case of a single $\mathrm{Cu}$ atom per cell, it is mostly the valence band part of the DOS that is affected.

\section{DISCUSSION AND CONCLUSIONS}

Using the $a b$ initio DFT method we have investigated the structural and electronic properties of narrow BC nanotubes. We have shown that infinite narrow $\mathrm{BC}$ tubes are energetically more stable than the corresponding strips of boron carbide hexagonal sheets. We have also found that isolated clean BC tubes keep their tubular shape and that their total energy decreases with increasing diameter of the tube. Both boron carbide hexagonal layers and clean $\mathrm{BC}$ tubes are metallic.

Doping has been shown to strongly affect the atomic and electronic properties of narrow $\mathrm{BC}$ tubes, with the effect depending on the diameter and helicity of the tubes, and the type of dopant atom. $(6,0)$ and $(4,4) \mathrm{BC}$ tubes doped with $\mathrm{Cu}$ and $\mathrm{Li}$ were determined to be metallic. Doping with $\mathrm{Li}$ has been shown to produce a tubular structure with the dopant atom positioned on the longitudinal axis for the $(6,0)$ tube, but with the dopant atom bonded to one side of the tubular shell for the $(4,4) \mathrm{BC}$ tube. We have found that increased doping with $\mathrm{Li}$ (up to three lithium atoms per unit cell) does not significantly change the shape of the $(6,0)$ BC tube. Doping with $\mathrm{Li}$ has also been found to shift the Fermi energy level, but no significant change in the DOS at this level has been observed. Calculations of the binding energy and heat of formation indicate that it would be difficult to achieve high concentrations of $\mathrm{Li}$ inside isolated, narrow $(6,0) \mathrm{BC}$ tubes. The bonding between the $\mathrm{Li}$ atoms and the boron carbon network has been determined to be of predominantly ionic character. It is the repulsive interactions between the $\mathrm{Li}$ ions that is believed to make the Li-doped BC tubes less stable at higher doping concentrations.
We have shown that doping narrow $\mathrm{BC}$ tubes with $\mathrm{Cu}$ leads to very different effects to those observed for $\mathrm{Li}$ dopant atoms. In contrast to $\mathrm{Li}, \mathrm{Cu}$ atoms inside a $(6,0) \mathrm{BC}$ tube are bonded to one side of the shell, while in the $(4,4) \mathrm{BC}$ tube they are located along the central longitudinal axis and give rise to a regular, rectangularlike cross section for the shell. This rectangularlike shape is retained as the concentration of $\mathrm{Cu}$ is increased. The electronic structures of the $(4,4)$ and $(6,0) \mathrm{BC}$ tubes both change significantly as a result of doping with $\mathrm{Cu}$, with the valence band DOS being greatly enhanced by contributions from the $\mathrm{Cu} d$ orbitals. Analysis of the charge density distribution and projected DOS reveals a predominantly covalent character for the bonding between the $d$ orbitals of the $\mathrm{Cu}$ atoms and the $\mathrm{C}$ atoms on the shell. Predominantly covalent bonds are also formed between the $\mathrm{Cu}$ atoms themselves. Our results show that the symmetric rectangularlike structure of the isolated $(4,4) \mathrm{BC}$ tube doped with higher concentrations of $\mathrm{Cu}$ is stabilized by the formation of these covalent bonds between the $\mathrm{Cu}$ dopant atoms. This is in contrast to the $(6,0)$ tubes doped with Li. These tubes acquire their cylindrical shape due to charge transfer from the Li atoms to the shell, but are destabilized by the repulsive interaction between the dopant atoms at high concentration.

In conclusion, we have characterized both clean and doped $\mathrm{BC}$ nanotubes and shown them to be viable structures. The stability of these tubes under heat or mechanical treatment, however, still remains a subject for further careful investigation.

\section{ACKNOWLEDGMENTS}

One of us (O.P.) would like to thank the University of Newcastle for financial support. We would also like to thank the APAC Supercomputing facility at the Australian National University for processing time and the Space Physics Group at the University of Newcastle for access to their SUN ULTRA-HPC450 computer.

\section{*Corresponding author.}

Email address: marian.radny@ newcastle.edu.au

${ }^{1}$ S. Iijima, Nature (London) 354, 56 (1991).

${ }^{2}$ A. Rubio, J. L. Corkill, and M. L. Cohen, Phys. Rev. B 49, 5081 (1994).

${ }^{3}$ Y. Miyamoto, A. Rubio, M. L. Cohen, and S. G. Louie, Phys. Rev. B 50, 4976 (1994).

${ }^{4}$ Z. Weng-Sieh, K. Cherrey, N. G. Chopra, X. Blase, Y. Miyamoto, A. Rubio, M. L. Cohen, S. G. Louie, A. Zettl, and R. Gronsky, Phys. Rev. B 51, 11229 (1995).

${ }^{5}$ W. Q. Han, P. Kohler-Redlich, T. Seeger, F. Ernst, M. Ruhle, N. Grobert, W. K. Hsu, B. H. Chang, Y. Q. Zhu, H. W. Kroto, D. R. M. Walton, M. Terrones, and H. Terrones, Appl. Phys. Lett. 77, 1807 (2000).

${ }^{6}$ S. M. Lee, Y. H. Lee, Y. G. Hwang, J. Elsner, D. Porezag, and T. Frauenheim, MRS Internet J. Nitride Semicond. Res. 4, art. no. G6.3 (1999).
${ }^{7}$ R. Tenne, L. Margulis, M. Genut, and G. Hodges, Nature (London) 380, 444 (1992).

${ }^{8}$ Y. R. Hacohen, E. Grunbaum, R. Tenne, J. Sloan, and J. L. Hutchison, Nature (London) 395, 336 (1998).

${ }^{9}$ B. Alperson, M. Homyonfer, and R. Tenne, J. Electroanal. Chem. 473, 186 (1999).

${ }^{10}$ B. Mayers and Y. N. Xia, Adv. Mater. (Weinheim, Ger.) 14, 279 (2002).

${ }^{11}$ Y. Li, J. Wang, Z. Deng, Y. Wu, X. Sun, D. Yu, and P. Yang, J. Am. Chem. Soc. 123, 9904 (2001).

${ }^{12}$ M. Nath, S. Kar, A. Raychaudhuri, and C. Rao, Chem. Phys. Lett. 368, 690 (2003).

${ }^{13}$ Z. Tao, S. Li, X. Fan, and J. Chen, Chin. J. Chem. 20, 99 (2004).

${ }^{14}$ R. Tenne and A. K. Zettl, in Carbon Nanotubes: Synthesis, Structure, Properties, and Applications, edited by M. S. Dresselhaus, G. Dresselhaus, and P. Avouris (Springer-Verlag, Berlin, 2001), Vol. 80, p. 81. 
${ }^{15}$ I. Boustani, A. Quandt, E. Hernandez et al., J. Chem. Phys. 110, 3176 (1999).

${ }^{16}$ V. Ivanovskaya, A. Enyashin, N. Medvedeva, and A. L. Ivanovskii, Phys. Status Solidi B 238, 1 (2003).

${ }^{17}$ V. Ivanovskaya, A. Enyashin, and A. Ivanovskii, Inorg. Mater. 40, 134 (2004).

${ }^{18}$ V. Ivanovskaya, A. Enjashin, A. Sofronov, Y. Makurin, N. Medvedeva, and A. Ivanovskii, J. Mol. Struct.: THEOCHEM 625, 9 (2003).

${ }^{19}$ I. Shein, V. Ivanovskaya, N. Medvedeva, and A. Ivanovskii, JETP Lett. 76, 189 (2002).

${ }^{20}$ G. Seifert and E. Hernandez, Chem. Phys. Lett. 318, 355 (2000).

${ }^{21}$ G. Seifert, T. Köhler, Z. Hajnal, and T. Frauenheim, Solid State Commun. 119, 653 (2001).

${ }^{22} \mathrm{G}$. Seifert, H. Terrones, M. Terrones, and T. Frauenheim, Solid State Commun. 115, 635 (2000).

${ }^{23}$ G. Seifert, T. Kohler, K. H. Urbassek, E. Hernandez, and T. Frauenheim, Phys. Rev. B 63, 193409 (2001).

${ }^{24}$ S. Gemming and G. Seifert, Phys. Rev. B 68, 075416 (2003).

${ }^{25}$ A. Quandt, A. Y. Liu, and I. Boustani, Phys. Rev. B 64, 125422 (2001).

${ }^{26}$ P. Zhang and V. H. Crespi, Phys. Rev. Lett. 89, 056403 (2002).

${ }^{27}$ A. Ivanovskii, Phys. Solid State 45, 1829 (2003).

${ }^{28}$ E. Montevecchi and J. O. Indekeu, Phys. Rev. B 62, 14359 (2000).

${ }^{29}$ H. Rosner, A. Kitaigorodsky, and W. E. Pickett, Phys. Rev. Lett. 88, 127001 (2002).

${ }^{30}$ M. J. Mehl, D. Papaconstantopoulos, and D. J. Singh, Phys. Rev. B 64, 140509(R) (2001).

${ }^{31}$ O. Ponomarenko, M. W. Radny, P. V. Smith, and G. Seifert, Phys. Rev. B 67, 125401 (2003).

${ }^{32}$ Z. Tang, N. Wang, X. Zhang, J. Wang, C. Chan, and P. Sheng, New J. Phys. 5, 146 (2003).

${ }^{33}$ M. Remskar, A. Mrzel, Z. Skraba, A. Jesih, M. Ceh, J. Demsar, P.
Stadelmann, F. Levy, and D. Mihailovic, Science 292, 479 (2001).

${ }^{34}$ G. Kresse and J. Hafner, Phys. Rev. B 47, 558 (1993); 49, 14251 (1994).

${ }^{35}$ G. Kresse and J. Furthmuller, Phys. Rev. B 54, 11169 (1996).

${ }^{36}$ J. P. Perdew and Y. Wang, Phys. Rev. B 45, 13244 (1992).

${ }^{37}$ G. Kresse and J. Hafner, J. Phys.: Condens. Matter 6, 8245 (1994).

${ }^{38} \mathrm{G}$. Kresse and J. Furthmuller, VASP the guide, in http:// cms.mpi.univie.ac.at/vasp/(2001).

${ }^{39}$ H. Monkhorst and J. Pack, Phys. Rev. B 13, 5188 (1976).

${ }^{40}$ T. Kar, J. Pattanayak, and S. Scheimer, J. Phys. Chem. A 105, 10397 (2001).

${ }^{41}$ E. Hernandez, C. Goze, P. Bernier, and A. Rubio, Phys. Rev. Lett. 80, 4502 (1998).

${ }^{42}$ G. G. Fuentes, E. Borowiak-Palen, M. Knupfer, T. Pichler, J. Fink, L. Wirtz, and A. Rubio, Phys. Rev. B 69, 245403 (2004).

${ }^{43}$ G. Lombardo, A. Grassi, G. Forte, G. Angilella, R. Pucci, and N. March, Phys. Lett. A 338, 303 (2005).

${ }^{44}$ C. Garau, A. Frontera, D. Quinonero, A. Costa, P. Ballestar, and P. Deya, Chem. Phys. 297, 85 (2004).

${ }^{45}$ Y. Yagi, T. M. Briere, M. H. F. Sluiter, A. A. Farajian, and Y. Kawazoe, Phys. Rev. B 69, 075414 (2004).

${ }^{46}$ J. Yang, H. J. Liu, and C. T. Chan, Phys. Rev. B 64, 085420 (2001).

${ }^{47}$ V. Meunier, J. Kephart, C. Roland, and J. Bernholc, Phys. Rev. Lett. 88, 075506 (2002).

${ }^{48}$ Z. Zhou, X. Gao, J. Yan, D. Song, and M. Morinaga, Carbon 42, 2677 (2004).

${ }^{49}$ Y. Miyamoto, A. Rubio, X. Blase, M. L. Cohen, and S. G. Louie, Phys. Rev. Lett. 74, 2993 (1995).

${ }^{50}$ A. Rubio, Y. Miyamoto, X. Blase, M. L. Cohen, and S. G. Louie, Phys. Rev. B 53, 4023 (1996). 